\title{
Three studies supporting the initial validation of the stress numerical rating scale-11 (Stress NRS-11): A single item measure of momentary stress for adolescents and adults
}

\author{
Dina Karvounides ${ }^{1,2 *}$, Pippa M. Simpson ${ }^{1,2}$, W. Hobart Davies ${ }^{3}$, Kim A. Khan ${ }^{1,2}$, Steven J. Weisman ${ }^{1,2}$ and Keri R. Hainsworth $^{1}$ \\ ${ }^{1}$ Medical College of Wisconsin, Milwaukee, Wisconsin, USA \\ ${ }^{2}$ Children's Hospital of Wisconsin, Milwaukee, Wisconsin, USA \\ ${ }^{3}$ University of Wisconsin-Milwaukee, Milwaukee, Wisconsin, USA
}

\begin{abstract}
Introduction: This study aimed to provide initial validation of the Stress Numerical Rating Scale-11 (SNRS-11) The validation is based on 3 different studies: Study A compared the SNRS-11 with the Pain Numerical Rating Scale-11 (PNRS-11), Spielberger's State Anxiety Inventory for Children (STAI-C-S), Perceived Stress Scale (PSS), and Children's Somatization Inventory (CSI) in 73 adolescents that were part of a larger study assessing physical and mental health benefits of yoga. Study B compared the SNRS-11 with thePNRS-11, Perceived Stress Scale (PSS), Liebowitz Social Anxiety Scale (LSAS), and 12-item Short Form Health Survey (SF12) in a community sample of 438 emerging adults. Study C compared the SNRS-11 with the Perceived Stress Scale (PSS), Pediatric Symptom Checklist-35 (PSC) and measures of physical health and family communication from a community sample of 171 parents/caretakers. Discussion: Findings suggest that the SNRS11 is a promising measure for efficiently assessing current stress in adolescents and adults.
\end{abstract}

Three studies supporting the initial validation of the Stress Numerical Rating Scale-11 (Stress NRS-11): A single item measure of momentary stress.

\section{Introduction}

For many years research has highlighted the effects of stress on one's physical and psychological health. For example, chronic stress can cause a lasting impact on the musculoskeletal, immune and inflammatory, cardiovascular, reproductive and gastrointestinal systems [1,2]. Specifically, stress has been linked to coronary heart disease, the progression of cancer, and diabetes [3-5]. Further, it can lead to mental health issues such as depression and anxiety $[1,2,6]$. Therefore, the assessment of stress for research and clinical purposes is extremely important.

Although very brief tools have been used to assess momentary discomfort, such as the Subjective Units of Discomfort Scale [7], no scale has measured momentary stress. In fact, Littman, White, Satia, Bowen, and Kristal [8] note that the assessment of stress has primarily focused on quantifying stressors or examining psychological responses to stressors. Very brief measures of stress have been validated in adults. For example, Littman and colleagues [8] developed 2 single-item measures of stress. The difficulty with this measure is that it does not capture momentary stress, and it assesses stress over the past year, which would be difficult for children. Validated subjective stress scales that are currently being used with pediatric populations include the Children's Hassles Scale (43 questions), the Perceived Stress Scale (14 questions; 10 questions for short version) and the Children's Hassles and Uplifts Scale (25 questions). Although each is a valuable tool, they can be lengthy to complete (10 to 43 questions), and with the exception of The Children's Hassles Scale, virtually all assess past stress to estimate current stress. Families might perceive the process as demanding and exhausting, especially if the measure is included as one of several measures and forms. Therefore the length of these measures may not be practical in a fast paced, hospital or clinical environment. Further, due to length, the mode of administration is limited to primarily paper and pencil or verbal communication. Taking into consideration the new wave of mobile health (mHealth) research, it would be difficult and cumbersome to administer multiple questions through most types of mHealth technology.

In addition to length and mode of administration, measures that require one's appraisal of past events or experiences can be subject to retrospective bias. Sato and Kawahara [9] note that when questionnaires depend on a respondent's retrospective memory, estimated mood states can be subject to issues of memory function. Specifically, studies have shown that explicit memory is reconstructive; therefore retrieval of past thoughts and feelings can be influenced by current thoughts and mood state [10].

One example of a measure that can be influenced by memory bias is the Perceived Stress Scale (PSS). The PSS is a measure of one's global life stressors within the last month. A respondent's rating of how often he or she has felt nervous and stressed within the past month could be subject to retrospective bias depending on his or her memory of past

Correspondence to: Dina Karvounides, Medical College of Wisconsin, Pediatric Headache Program, Children's Hospital of Philadelphia, 3501 Civic Center Boulevard, PA 19104, Philadelphia, USA, Tel: (267) 426-8337; Fax: (215) 5902223; E-mail: karvounidd@email.chop.edu

Key words: stress, validation, adolescents, pain

Received: June 28, 2016; Accepted: July 27, 2016; Published: July 30, 2016 
events and current emotional state. Although the PSS has been used as a measure of perceived stress in the past month, the stress hormone glucocorticoid can significantly impair retrieval of past events [11,12]. Therefore if one were to feel stressed at the time of completing a measure such as the PSS, it is likely that increased glucocorticoid levels could interfere with an accurate completion of the measure.

Overall, currently used subjective stress scales have disadvantages such as length and mode of administration as well as potential retrospective bias, in addition to the difficulty of assessing past stress to estimate current stress. Thus, a brief measure of current stress that is easily administered, amenable to multiple modes of administration and utilizes a brief screening of current stress level would be a useful clinical and research tool. To this end, we developed the Stress Numerical Rating Scale-11 (SNRS-11).

The SNRS-11 was modeled after the commonly used single point assessment of pain, the NRS-11 [13]. Like the NRS-11, the SNRS-11, requires participants to respond to the question, "On a scale of 0 to 10 , with 0 being no stress and 10 being worst stress possible, what number best describes your level of stress right now?" Below the question, the 11 numbers were spaced equally across the page, with the anchors noted below the " 0 " and the " 10 ." Participants respond by circling the number corresponding to their current level of stress. The SNRS-11 also focuses on stress intensity, which is one dimension of the multidimensional construct of stress, similar to the NRS-11 that focuses on the assessment and measurement of pain intensity, one dimension of pain. The pain NRS- 11 has been validated in studies with adults and children as young as 8 years old $[13,14]$. Specifically, the pain NRS-11 has been validated against the Faces Pain Scale-Revised (FPS-R; 69 children age 7-17 years), the Visual Analogue Scale (VAS; 29 children age 9-17 years) and against the VAS with a large sample $(n=236)$ of children [13]. Use of the pain NRS-11 requires children to rate "...how much pain she or he has, using a number from 0 to 10 where 0 is no pain or hurt and 10 is the most or worst pain" [13]. In these studies, the pain NRS11 was found to be "functionally equivalent" to the other pain scales, and was also considered to have several important advantages over the other scales. The objective of the present study was to provide initial validation of the SNRS-11 in three separate, unpublished studies.

\section{Study A: the SNRS-11 and STAI-C-S, PSS, CSI in high school adolescents}

\section{Method}

Participants were part of a larger study assessing the physical and mental health benefits of yoga for sophomore students at an urban, public high school. Based on data from the most recent census (2010-2011), this high school is comprised of approximately 1,610 total students; $51 \%$ female, 69\% African American, 14\% Hispanic, $10 \%$ Caucasian, $8 \%$ Asian, $1 \%$ Native American and $75 \%$ of students receive free/reduced lunch. Of the 118 students approached in the combined classes, 73 consented to participate. Participants were 14 to 17 years old with a mean age of $15.0(S D=.70)$ years. The sample was representative of the larger school population: the majority of students were female $(62.5 \%)$, and the sample was comprised of a racially diverse group of students with $62.0 \%$ African-American, $14.1 \%$ Mixed Race, 11.3\% Asian, 11.3\% Hispanic, and 1.4\% Eastern Indian. All students enrolled in the 2 participating physical education classes were eligible for the study. Parental consent and student assent were required for participation. This study utilized a modified cross over design. Students were randomized to either the yoga or the control group (2 times per week), allowing for comparison of yoga practice against traditional strength training in the school's wellness center. The first class continued this split for the entire semester (22 classes), whereas the second class switched conditions at mid-semester.

For the larger study, the SNRS-11 and study questionnaires were administered pre- (at the start of each semester), mid- and postintervention. In addition, participants rated stress and pain immediately after each yoga/wellness class. As stress is a transient construct, the daily ratings were collapsed to calculate a mean daily stress or pain score for each participant (reported below as "mean daily stress scores" and "mean daily pain scores," respectively). As the PSS assesses stress over the past month, we chose to use the daily stress ratings collapsed across the first half of the study ( $\mathrm{n}=11$ classes) and the questionnaire data collected at the study mid-point. This study was approved by the Institutional Review Board of the Medical College of Wisconsin and by the Research Division of the Milwaukee Public Schools. Measures included the SNRS-11, Spielberger's State-Trait Anxiety Inventory for Children (STAI-C), the Perceived Stress Scale (PSS), the Children's Somatization Inventory (CSI). Participants also rated current pain severity using the Pain Numerical Rating Scale-11.

\section{Results}

Prior to conducting analyses, data from the 4 separate classes ( 2 classes X 2 semesters) was examined to determine if the classes differed on critical demographic variables. No differences were found for ethnicity or gender; therefore, data was collapsed and used as a single dataset for all analyses (all $\mathrm{p}$ values $>.05$ ). The psychometric properties for each measure are presented in Table 1. Overall, scores on the SNRS-11 suggest that the sample reported low levels of stress, with $75 \%$ of the scores falling below 3.75 on the scale. A bubble plot (Figure 1) illustrates ratings on the SNRS-11 plotted against question 3 of the PSS: "In the last month, how often have you felt nervous and stressed?" Although stress ratings were low in general on both scales, the plot illustrates general commonality between ratings. As expected, the correlation between the SNRS-11 and the STAI-C, our primary measure of construct validity was strong: $r_{s}=.60, p<.01$. The SNRS11 was also significantly and moderately correlated with both the PSS Total score: $\left(r_{\mathrm{s}}=.31 p<.01\right)$ and the CSI-24 Total score $\left(r_{\mathrm{s}}=.35\right.$, $\mathrm{p}<.01)$. The SNRS-11 also indicated a strong relationship with current usual pain: $r_{\mathrm{s}}=.57, p<.01$. Current SNRS-11 scores were moderately correlated with students' mean daily stress scores $\left(r_{\mathrm{s}}=.41, \mathrm{p}<.01\right)$. Further, mean daily SNRS-11 scores reported over the first half of the semester were strongly correlated with mean daily pain scores averaged over the past two months (NRS-11) reported over the same timeframe $\left(r_{s}=.68, p<.01\right)$. In our sample, females reported significantly higher $(p<.05)$ stress on the SNRS-11 $(3.0,0.00-5.00)$ than males $(1.00,0.00$ $2.00)$. However, there were no differences in SNRS-11 scores for youth involved in the yoga vs. wellness groups $(p>.05)$.

Table 1. Descriptive data for all measures.
\begin{tabular}{|l|c|c|c|c|c|}
\hline Measure & Median & IQR & Mean & SD & Range \\
\hline SNRS-11 & 2.00 & $0.00-3.75$ & 2.5 & 2.8 & $0-10$ \\
\hline $\begin{array}{l}\text { (0-10) } \\
\text { Female } \\
\text { Male }\end{array}$ & 3.00 & $0.00-5.00$ & & & \\
\hline STAI-C & 1.00 & $0.00-2.00$ & & & \\
\hline $\begin{array}{l}(0-60) \\
\text { PSS } \\
(0-56)\end{array}$ & 32.0 & $28.0-36.0$ & 32.2 & 5.8 & $20-45$ \\
\hline $\begin{array}{l}\text { CSI-24 } \\
(0-140)\end{array}$ & 16.0 & $11.0-20.0$ & 16.0 & 6.9 & $2-33$ \\
\hline
\end{tabular}




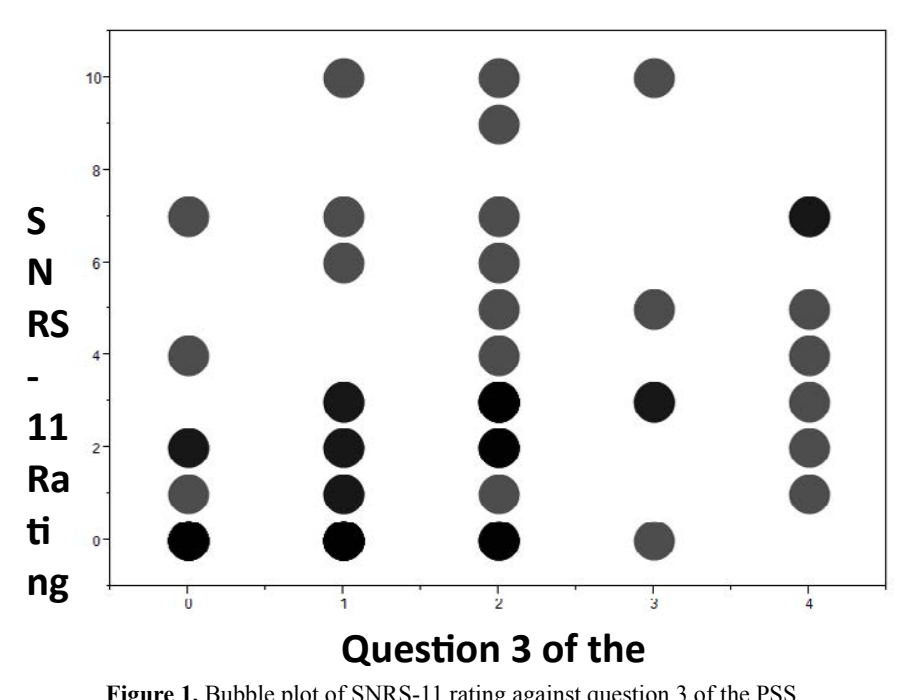

Figure 1. Bubble plot of SNRS-11 rating against question 3 of the PSS.

Study B: the SNRS-11 with the Pain Numeric Rating Scale-11, PS, LSAS, and SF12 in a community sample of emerging adults

\section{Method}

Participants were part of a larger study assessing. The sample included 438 emerging adults (76\% students; $72 \%$ Caucasian), 18 24 years old (mean age 21) of whom 289 (66\%) were female. Out of the entire sample, $25 \%(n=104)$ reported experiencing chronic or recurrent pain. The SNRS-11 was compared with the Pain Numeric Rating Scale-11, Perceived Stress Scale (PSS), Liebowitz Social Anxiety Scale (LSAS), and 12-item Short Form Health Survey (SF12).

\section{Results}

When the SNRS-11 was compared to the SF-12, physical functioning was correlated with lowest stress over the past week $(\mathrm{r}=$ $.15, p<.01)$. General Health was correlated with highest stress over the past week $(\mathrm{r}=-.12, p<.05)$. As support for the validity of the SNRS-11, the strongest correlations were found for average mental health $\left(r_{s}=\right.$ $.51 p<.01)$ and emotional functioning $\left(r_{\mathrm{s}}=.34 p<.01\right)$ subscale scores from the SF-12. Those who reported having chronic or recurrent pain in the past also reported greater Current, Average, Highest and Lowest stress over the past week (all $p<.01$ ).

\section{Study C: Study C compared the SNRS-11 with the PSS, PSC-35 and measures of physical health and family communication from a community sample of parents/ caretakers}

\section{Method}

As part of a larger study utilizing a snowball sampling method, parents/caretakers of young children completed the SNRS-11, Perceived Stress Scale (PSS), Pediatric Symptom Checklist-35 (PSC) and measures of physical health and family communication. This community sample consisted of 171 parents/caretakers (73.5\% mothers), 16-48 years old (mean age 37 ) of whom $75 \%$ were Caucasian. Out of the entire sample, $57 \%$ responded about a male child ( $\mathrm{M}=9.4$, range: 6-12 years).

\section{Results}

Parents who reported having a difficult discussion with their child over the past two weeks reported greater Average and Highest stress over the past week $(\mathrm{p}<.05)$. Parents of child with a chronic illness reported greater stress over the past week $(\mathrm{p}<.05)$. Correlations of the SNRS-11 with the PSS and Pain NRS-11 were $r_{\mathrm{s}}=.49(p<.01)$ and $r_{\mathrm{s}}=$ $.29(p<.01)$ respectively.

\section{Discussion}

The aim of this study was to provide initial validation of a brief measure of current stress, the Stress Numerical Rating Scale - 11 (SNRS-11). Using well-validated measures for comparison, our scale showed moderate to strong construct validity and moderate concurrent validity. In Study A, as hypothesized, the SNRS-11 was strongly correlated with the STAI-C, a measure of current anxiety, and moderately correlated with the PSS and CSI-24, measures of stress and somatization over the past month, respectively. The SNRS-11 showed adequate construct validity from the mental health scale of the SF-12 in Study B as well as good discriminate and convergent validity with the PSS measure used in Study C. These results suggest that the SNRS-11 is a promising measure for the efficient assessment of current stress, particularly when the assessment of context-dependent stress may be important. These results are the first to report on a single-item stress measure for a pediatric sample.

The strength of the correlations of the SNRS-11 scores with the validated measures used in the current study is comparable to those found in similar studies. For example, [8] developed 2 single-item assessments of stress over the past year. One of their items was "In the past year, how would you rate the amount of stress in your life (at home and at work)?" When this item was compared against the hassles scale (a 53-item assessment of everyday stress), the adjusted correlation was .44, when compared against the 4 -item PSS the correlation was .34 , and when compared against the life events questionnaire (a 10 item measure of major life events), the correlation was .34. In the current study, the correlation between the SNRS-11 and the PSS was .31 which is similar to that described for the single item measure and the 4 -item PSS found by Littman et al. The strength of the relationship between the SNRS-11 and the STAI-C-S $\left(r_{s}=.60\right)$ was stronger than any of the relationships found by Littman et al., yet comparable to results found in the following study. Crandall, Lammers, Senders, [15], evaluated the validity of a single, self-report numeric zero to 10 scale used to measure children's (7-13 years old) state anxiety before and after preoperative education regarding their upcoming tonsillectomy and/or adenoidectomy at a university children's hospital ENT clinic. The authors examined the relationship between their numeric scale of anxiety against item 20 on the STAI-C ("How I feel right now"). The nonparametric correlation preeducation was 0.42 and posteducation was 0.64 . Overall, our results are consistent with other similar studies.

\section{Limitations}

There are multiple limitations that have surfaced in this study. One unusual finding that warrants further discussion is that the population of Study A reported lower levels of stress than expected. The majority of the population in this study is of racial minority status, receives free/ reduced lunches and resides in impoverished areas within the inner city. Research has found that adolescents who live in urban environments with racial and ethnic minority status are particularly vulnerable to stress $[16,17]$. and are subject to markedly different experiences that are often more stressful than those experienced by adolescents in nonurban, middle-class settings $[18,19]$. Given these findings, it was surprising to find that participants in this study reported stress levels 
Karvounides D (2016) Three studies supporting the initial validation of the stress numerical rating scale-11 (Stress NRS-11): A single item measure of momentary stress for adolescents and adults

on the lower end of the SNRS-11 and on the PSS scales. Indeed, the mean PSS score for participants in our study (16) was lower than that reported for Caucasian high school students in rural Boston (19.2) [20]. Overall, our findings suggest that further validation with other populations, including other age groups is necessary [21-25].

In addition to the above findings, the SNRS-11 is subject to social, cognitive, and contextual influences, similar to any other subjective rating scale. This scale was administered to a diverse population of sophomore students without controlling for intellectual capacity [26-28]. It is possible that students may have had a difficult time comprehending the battery of measures provided. However, student questions were addressed at the time of administration by the investigators. Students may have also different ways of operationalizing the word "stress" as this is a complex construct [29,30]. Further, level of motivation and commitment may have been influenced by the larger study goals (i.e. perceptions of the yoga intervention). Each student's report of momentary stress on the SNRS-11 may have also been influenced by the class period of being in gym class where stress is typically minimal compared to other more intense classes. Future studies could focus more specifically on contextual factors that might influence one's rating of momentary stress at the time of administration [31-33].

\section{Conclusions}

Overall, the results of this study suggest that the SNRS-11 is a promising tool for the assessment of current stress. It has practical advantages over other measures as it is brief and can be easily administered with paper and pencil, verbally, or through the use of mhealth technology. Like the Pain NRS, the SNRS is a subjective rating scale that can be easily used to track stress level over time. It can also be well utilized in conjunction with the Pain NRS.

Future studies should evaluate the SNRS-11 with other populations and medical conditions. This should include conditions in which respondents report stress levels at the upper end of the scale range. To assess the practical utility of the SNRS-11 as well as expanding the data on construct validity, future studies should evaluate the measure in other contexts. One example would be to determine whether momentary stress as rated with the SNRS-11 influences one's ability to process important medical information, such as at hospital discharge.

\section{Acknowledgements}

The authors thank Ashley A. Jacobson for her help with the study. We also thank Pam Kutcher and Paul Zettel for their help in facilitating this study.

This work was supported by 2 separate grants from the Children's Hospital of Wisconsin Medical Staff "Improving Health in Kids" fund to KRH.

\section{References}

1. Davis M, Eshelman E, McKay M (2008) The relaxation and stress reduction workbook. Oakland, CA: New Harbinger Publications.

2. Rice P (1999) Stress and health: Third edition. Pacific Grove, CA: Brooks/Cole Publishing Company.

3. Aikens JE, Wallander JL, Bell DS, McNorton A (1994) A nomothetic-idiographic study of daily psychological stress and blood glucose in women with type I diabetes mellitus. Journal of Behavioral Medicine 17: 535-548. [Crossref]

4. Li X, Lv Y, Yuan A, Li Z (2010) Gastrin-releasing peptide links stressor to cancer progression. Journal of Cancer Research and Clinical Oncology 136: 483-491. [Crossref]
5. Schreier NK, Moltchanova EV, Blomstedt PA, Kajantie E, Eriksson JG, et al. (2011) Prenatal exposure to wartime stress: Long-term effect on coronary heart disease in later life. Annals of Medicine 43: 555-561. [Crossref]

6. McEwen BS (2008) Central effects of stress hormones in health and disease Understanding protective and damaging effects of stress and stress mediators. European Journal of Pharmacology 583: 174-185. [Crossref]

7. Wolpe J (1969) The practice of behavior therapy (1st ed.) New York: Pergamon.

8. Littman AJ, White E, Satia JA, Bowen DJ, Kristal AR, et al. (2006) Reliability and Validity of 2 Single-Item Measures of Psychosocial Stress. Epidemiology 17: 398-403. [Crossref]

9. Sato H, Kawahara J (2011) Selective bias in retrospective self-reports of negative mood states. Anxiety, Stress, \& Coping 24: 359-367. [Crossref]

10. Wilson TD, Meyers J, Gilbert DT (2003) How happy was I, anyway? A retrospective impact bias. The Practice of Behavior Therapy 21: 421-446.

11. Cohen S, Kamarck T, Mermelstein R (1983) A global measure of perceived stress Journal of Health and Social Behavior 24: 385-396. [Crossref]

12. Lupien SJ, Maheu F, Tu M, Fiocco A, Schramek TE (2007) The effects of stress and stress hormones on human cognition: Implications for the field of brain and cognition. Brain and Cognition 65: 209-237. [Crossref]

13. von Baeyer CL, Spagrud LJ, McCormick JC, Choo E, Neville K, et al. (2009) Three new datasets supporting use of the numerical rating scale (NRS-11) for children's selfreports of pain intensity. Pain 143: 223-227. [Crossref]

14. Miró J, Castarlenas E, Huguet A (2009) Evidence for the use of a numerical rating scale to assess the intensity of pediatric pain. European Journal of Pain 13: 1089-1095. [Crossref]

15. Crandall M, Lammers C, Senders C, Savedra M, Braun JV, et al. (2007) Initial validation of a numeric zero to ten scale to measure children's state anxiety. Anesthesia and Analgesia 105: 1250-1253. [Crossref]

16. Choi H, Meininger JC, Roberts RE (2006) Ethnic differences in adolescent's mental distress, social stress, and resources. Adolescence 41: 263. [Crossref]

17. Vacek KR, Coyle LD, Vera EM (2010) Stress, self-esteem, hope, optimism, and wellbeing in urban, ethnic minority adolescents. Journal of Multicultural Counseling and Development 38: 99-111.

18. Lynam DR, Caspi A, Moffitt TE, Wikström PO, Loeber R, et al. (2000) The interaction between impulsivity and neighborhood context on offending: The effects of impulsivity are stronger in poorer neighborhoods. Journal of Abnormal Psychology 109: 563-574 [Crossref]

19. Schmeelk-Cone K H, Zimmerman MA (2003) A longitudinal analysis of stress in african american youth: Predictors and outcomes of stress trajectories. Journal of Youth and Adolescence 32: 419-430.

20. Noggle JJ, Steiner NJ, Minami T, Khalsa SB (2012) Benefits of yoga for psychosocial well-being in a U.S. high school curriculum: A preliminary randomized controlled trial. Journal of Developmental and Behavioral Pediatrics 33: 193-201. [Crossref]

21. Cohen S, Williamson GM (1988) Perceived stress in a probability sample of the united states. In S. Spacapan, \& S. Oskamp (Eds.) The social psychology of health. Newbury Park, CA: Sage.

22. Cross R, Huberty T (1993) Factor analysis of the state-trait anxiety inventory for children with a sample of seventh- and eigth-grade students. Journal of Psychoeducational Assessment 11: 232-241.

23. Egger HL, Costello EJ, Erkanli A, Angold A (1999) Somatic complaints and psychopathology in children and adolescents: Stomach aches, musculoskeletal pains, and headaches. Journal of the American Academy of Child and Adolescent Psychiatry 38: 852-860. [Crossref]

24. Garber J, Walker LS, Zeman J (1991) Somatization symptoms in a community sample of children and adolescents: Further validation of the children's somatization inventory Psychological Assessment 3: 588-595.

25. Golden-Kreutz DM, Browne MW, Frierson GM, Andersen BL (2004) Assessing stres in cancer patients: A second-order factor analysis model for the perceived stress scale. Assessment 11: 216. [Crossref]

26. Sibinga EM, Kerrigan D, Stewart M, Johnson K, Magyari T, et al. (2011) Mindfulnessbased stress reduction for urban youth. Journal of Alternative and Complementary Medicine (New York, N.Y.), 17: 213-218. [Crossref] 
Karvounides D (2016) Three studies supporting the initial validation of the stress numerical rating scale-11 (Stress NRS-11): A single item measure of momentary stress for adolescents and adults

27. Spielberger CD (1973) Stait-trait anxiety inventory for children. Menlo Park, CA: Mind Garden, Inc.

28. Vila M, Kramer T, Hickey N, Dattani M, Jefferis H, et al. (2009) Assessment of somatic symptoms in british secondary school children using the children's somatization inventory (CSI). Journal of Pediatric Psychology 34: 989-998. [Crossref]

29. Walker LS, Garber J, Greene JW (1991) Somatization symptoms in pediatric abdominal pain patients: Relation to chronicity of abdominal pain and parent somatization. Journal of Abnormal Child Psychology 19: 379-394. [Crossref]

30. Walker LS, Beck JE, Garber J, Lambert W (2009) Children's somatization inventory:
Psychometric properties of the revised form (CSI-24). Journal of Pediatric Psychology 34: 430-440. [Crossref]

31. Liebowitz MR (1987) Social phobia. Mod. Probl. Pharmacopsychiatry 22: 141-173.

32. Littman AJ, White E, Satia JA, Bowen DJ, Kristal AR, et al. (2006) Reliability and Validity of 2 Single-Item Measures of Psychosocial Stress. Epidemiology 17: 398-403. [Crossref]

33. Ware J Jr, Kosinski M, Keller SD (1996) A 12-Item Short-Form Health Survey: construction of scales and preliminary tests of reliability and validity. Medical Care 34: 220-33. [Crossref]

Copyright: (C2016 Karvounides D. This is an open-access article distributed under the terms of the Creative Commons Attribution License, which permits unrestricted use, distribution, and reproduction in any medium, provided the original author and source are credited. 